\title{
Efficacy and Safety of Pitavastatin in a Real-World Setting: Observational Study Evaluating SaFety in Patient Treated with Pitavastatin in Korea (PROOF Study)
}

\author{
In-Kyung Jeong ${ }^{1}$, Sung-Rae Kim²
}

${ }^{1}$ Department of Endocrinology, Kyung Hee University Hospital at Gangdong, Kyung Hee University School of Medicine, Seoul; ${ }^{2}$ Division of Endocrinology and Metabolism, Department of Internal Medicine, Bucheon St. Mary's Hospital, College of Medicine, The Catholic University of Korea, Seoul, Korea

Background: While randomized controlled trials provide useful information about drug safety and efficacy, they do not always reflect the observed results in the real world. The prospective, observational, non-comparative trial in South Korea was designed to evaluate the efficacy and safety of pitavastatin in clinical practice in 28,343 patients.

Methods: This study was conducted in 893 facilities in Korea from April 2, 2012 to April 1, 2017. This study was designed to administer 1, 2, or $4 \mathrm{mg}$ pitavastatin to patients with hyperlipidemia at the age of 20 or older for at least 8 weeks.

Results: For 126 days of mean duration of administration of pitavastatin, the $\%$ change of low density lipoprotein cholesterol indicated a dose dependent reduction: $-23.4 \%,-29.1 \%$, and $-35.2 \%$ in the 1,2 , and $4 \mathrm{mg}$ groups, respectively in patients who have not been treated with lipid lowering medications prior to study. Only $1.74 \%(492 / 28,343)$ of pitavastatin-treated patients experienced adverse events, of which $0.43 \%(123 / 28,343)$ were adverse drug reactions. Less than $1 \%$ of patients experienced the grade 2 or more toxicity (Common Terminology Criteria for Adverse Events v4.03) in alanine aminotransferase, aspartate aminotransferase, serum creatinine, and serum creatine phosphokinase. Although there were no rhabdomyolysis in 28,343 patients, $0.04 \%$ of patients had been reported pitavastatin-related musculoskeletal disorders.

Conclusion: Overall, this observational study showed that pitavastatin was well tolerated and effectively modified the lipid profile, reducing cardiovascular and cerebrovascular risk in Korean patients with hypercholesterolemia in the real world.

Keywords: Pitavastatin; Observational study; Cholesterol, LDL; Safety; Rhabdomyolysis

\section{INTRODUCTION}

Cardiovascular disease (CVD) has been prevalent worldwide and is one of the leading causes of death [1]. Comprehensive management of cardiovascular risk factors such as dyslipid- emia, hypertension, diabetes mellitus (DM), and smoking is recommended. Since the prevalence of hypercholesterolemia has risen consistently increased, many guidelines recommended statin as the first drug to prevent CVD according to the CVD risk and low density lipoprotein cholesterol (LDL-C) level [2].
Received: 27 May 2020, Revised: 2 September 2020,

Accepted: 10 September 2020

Corresponding author: Sung-Rae Kim

Division of Endocrinology and Metabolism, Department of Internal Medicine, Bucheon St. Mary's Hospital, College of Medicine, The Catholic University of Korea, 327 Sosa-ro, Wonmi-gu, Bucheon 14647, Korea

Tel: +82-32-340-7013, Fax: +82-32-340-2039, E-mail: kimsungrae@catholic.ac.kr

\section{Copyright (C) 2020 Korean Endocrine Society}

This is an Open Access article distributed under the terms of the Creative Commons Attribution Non-Commercial License (https://creativecommons.org/ licenses/by-nc/4.0/) which permits unrestricted non-commercial use, distribution, and reproduction in any medium, provided the original work is properly cited. 
Statins, such as atorvastatin, rosuvastatin, simvastatin, and pitavastatin, also known as 3-hydroxy-3-methylglutaryl coenzyme A (HMG-CoA) reductase inhibitors, are one of the most frequently prescribed drugs to control hypercholesterolemia in recently growing patients with dyslipidemia and ischemic heart diseases. These HMG-CoA reductase inhibitors are proven not only to reduce total cholesterol, LDL-C, and triglyceride in blood but also to increase high density lipoprotein cholesterol (HDL-C) $[3,4]$.

In a number of epidemiology studies, statin treatment was proven to reduce LDL-C, thereby preventing CVD. In terms of the LDL-C lowering effect, pitavastatin $2 \mathrm{mg}$ produced significantly greater reductions than simvastatin $20 \mathrm{mg}$ after 12 weeks of treatment ( $-39 \%$ vs. $-35 \%, P=0.014$, respectively), as a result of a phase 3 randomized controlled trial (RCT) in 857 Caucasian patients [5]. The results of an observational study confirming the effect of reducing LDL-C when various types of statins were administered in 909 Koreans who needed statin administration were: Atorvastatin $10 \mathrm{mg}$, lovastatin $20 \mathrm{mg}$, pitavastatin $2 \mathrm{mg}$, pravastatin $20 \mathrm{mg}$, rosuvastatin $5 \mathrm{mg}$, and simvastatin $20 \mathrm{mg}$ significantly reduced LDL-C by $41.8 \% \pm$ $11.0 \%, 33.8 \% \pm 12.8 \%, 39.3 \% \pm 10.8 \%, 31.5 \% \pm 8.9 \%, 48.8 \% \pm$ $12.3 \%$, and $42.8 \% \pm 13.5 \%$, respectively [6]. In the results of a multicenter, randomized trial in 268 Koreans, there were no significant differences between the pitavastatin and atorvastatin groups in terms of the mean \pm standard deviation (SD) percent change in LDL-C at the end of the study $(-42.9 \pm 12.7 \mathrm{mg} / \mathrm{dL}$ vs. $-44.1 \pm 11.1 \mathrm{mg} / \mathrm{dL}$ ) [7].

Regarding the pitavastatin's CVD outcome, The Randomized Evaluation of Aggressive or Moderate Lipid Lowering Therapy with Pitavastatin in Coronary Artery Disease (REAL-CAD) study has demonstrated the effectiveness and safety of pitavastatin in CVD. Results from the comparison of cardiovascular death, nonfatal myocardial infarction, nonfatal ischemic stroke, and hospitalization for unstable angina between the pitavastatin $1 \mathrm{mg} /$ day $(n=6,528)$ and $4 \mathrm{mg} /$ day $(n=6,526)$ groups in 13,054 Japanese patients with stable coronary artery disease showed a significantly greater decrease in the incidence of the protocol defined events in the $4 \mathrm{mg}$ /day group than in the $1 \mathrm{mg}$ /day group (266 patients [4.3\%] and 334 patients [5.4\%]; hazard ratio, $0.81 ; 95 \%$ confidence interval, 0.69 to $0.95 ; P=0.01$ ) [8].

Despite their favorable effects on CVD, statins are also associated with rare adverse effects such as abnormal hepatic function, myopathy, and newly developed DM [9]. In addition, statins are reported to potentially increase the risk of rhabdomyolysis in patients with hypothyroidism, in the elderly, or in those concomitantly treated with fibrates or drugs metabolized by CYP450 [10-12]. Generation of the muscular membrane and change of its component as well as reduced production of Coenzyme-Q are known to contribute to the development of rhabdomyolysis although the exact underlying mechanism is yet to be discovered. As a matter of fact, impaired mitochondria was found in patients with rhabdomyolysis reported after rosuvastatin treatment [13]. Statins are contraindicated for use in patients with a history of myopathy. In the precautions for atorvastatin use, it is specified in the musculoskeletal impacts section that $0.4 \%$ of patients treated with atorvastatin in a clinical trial reported $\geq 10$ folds the upper normal limit of creatine phosphokinase (CPK), with $0.1 \%$ of these patients experiencing myalgia, sensitivity, or weakness [14].

As part of the risk management plan for pitavastatin (Livalo, JW Pharmaceutical Corporation, Seoul, Korea), a large-scale safety study titled the LIVALO Effectiveness and Safety (LIVES) Study was conducted in Japan. In this study, elevated blood CPK and rhabdomyolysis were reported in $2.74 \%$ (545/19,925 subjects) and $0.01 \%(2 / 19,925$ subjects), respectively $[15,16]$.

In light of these findings, the efficacy and safety of Pitavastatin in a Real-wOrld setting: Observational study evaluating saFety in patient with pitavastatin for lipid lowering therapy in Korea (PROOF) study, a large-scale observational study in Koreans, was aimed to enroll 30,000 subjects in order to actively collect and address the safety information with pitavastatin on rhabdomyolysis that occurs very rarely but can be serious.

\section{METHODS}

\section{Study design and patient recruitment}

This prospective, observational, multi-center study was conducted in 893 facilities in Korea from April 2, 2012 to April 1, 2017 (for approximately 5 years). This study was designed to administer 1, 2, or $4 \mathrm{mg}$ pitavastatin to patients with hyperlipidemia at the age of 20 or older for at least 8 weeks. The dosage of pitavastatin to be administered was chosen at the judgment of the physician. It was reviewed and approved by established Institutional Review Boards (Hallym University Kangnam Sacred Heart Hospital IRB No. 2015-02-18, etc.), and all study patients voluntarily signed a written informed consent.

\section{Sample size}

The sample size was calculated based on the results of previous pitavastatin studies. In the LIVES study, a large-scale safety 
study for pitavastatin conducted in Japan, adverse drug reactions (ADRs) of which causal relationship to pitavastatin cannot be ruled out occurred in $10.38 \%$ (2,069/19,925 subjects); rhabdomyolysis and abnormal hepatic function occurred in $0.01 \%$ (2/19,925 subjects) and $0.03 \%(6 / 19,925$ subjects), respectively [17].

In the present study, the number of subjects required to detect the incidence of rhabdomyolysis with a $\geq 95 \%$ confidence level was calculated by using the following formula.

$$
\begin{aligned}
& \mathrm{N} \geq \frac{-\ln (1-0.95)}{\lambda}=\frac{-\ln (0.05)}{0.0001}=29,957.3 \\
& (\lambda=\text { incidence of adverse events [AEs] })
\end{aligned}
$$

\section{Patient data collection}

Subject information including demographics, health information, medical history, and previous treatment history was collected through medical examination on the start day of study treatment. The primary endpoint of this study was safety; biochemical markers including serum creatinine, alanine aminotransferase (ALT), aspartate aminotransferase (AST), and serum CPK were measured before and after treatment for comparative analysis. The secondary endpoint of this study was efficacy. For efficacy assessment, serum lipid profiles including total cholesterol, LDL-C, HDL-C, and triglyceride were measured before and after treatment for comparative analysis. Efficacy was assessed at least once during the study period.

\section{Statistical analysis}

Mean and SD were provided for continuous variables, while frequency (n) and percentage (\%) were presented for categorical variables. Categorical variables were analyzed using Pearson's chi-square test or Fisher's exact test. For comparing between two groups of continuous variable, 2-sample independent $t$ test was used when the data were normally distributed and Wilcoxon rank sum test was used if they were not. In addition, when comparing before and after treatment in the group, paired $t$ test was used if data were normally distributed and Wilcoxon signed rank test was used if they were not.

\section{RESULTS}

\section{Study subjects}

A total of 29,137 subjects were enrolled. Of those, 719 subjects not assessed for safety, 26 subjects used pitavastatin before the beginning of this study, 23 subjects with violations of the inclusion/exclusion criteria, 15 overlapped subjects, and 11 subjects

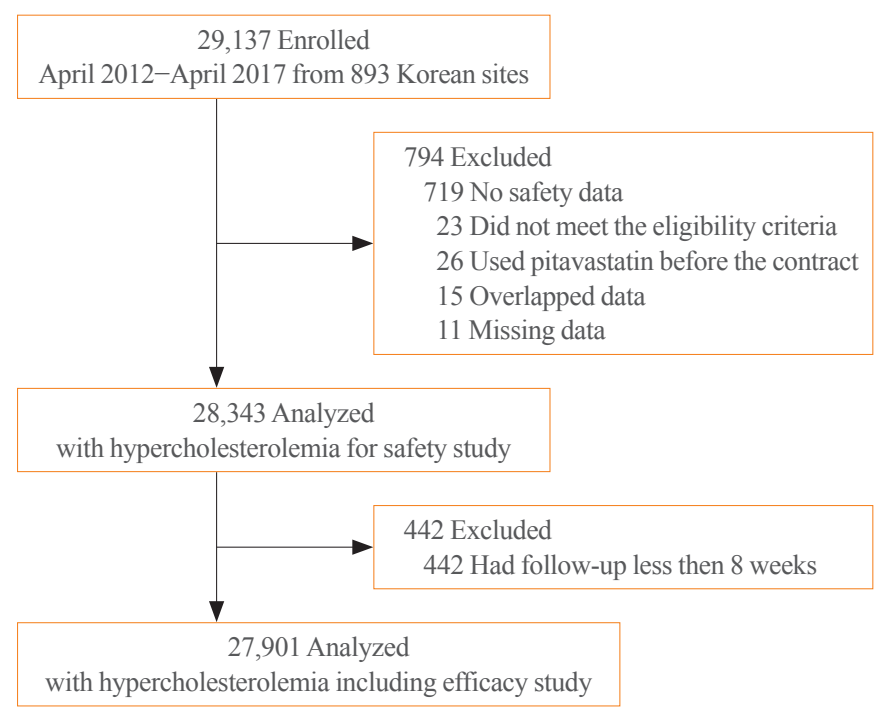

Fig. 1. Disposition of patients.

with missing data were excluded from the safety set; the safety results of pitavastatin were obtained from the remaining 28,343 subjects with hyperlipidemia. Of those, 442 subjects who were followed up for less than 8 weeks were excluded from the efficacy set; the efficacy results were obtained from the remaining 27,901 subjects (Fig. 1).

\section{Baseline clinical characteristics of subjects}

Table 1 showed the baseline characteristics of the study subjects. The elderly more than 65 years of age accounted for $38.1 \%$, and $36.7 \%$ of patients had been treated with lipid lowering medications prior to study. The percentage of patients with liver disease and renal disease was $4.4 \%$ and $3.3 \%$, respectively. In addition, $67.4 \%$ of patients had comorbidities: DM in $18.9 \%$ and hypertension in $38.9 \%$. The percentage of patients suffering from cardiovascular and cerebrovascular diseases was $10.7 \%$ and $11.9 \%$, respectively (Table 2).

\section{Administration of pitavastatin}

The mean duration of pitavastatin administration was $125.85 \pm$ 83.78 days. The duration of administration was 8 weeks to less than 16 weeks in $56.1 \%$ versus more than 24 weeks in $19.8 \%$. The dose of pitavastatin administered was $2 \mathrm{mg}$ in $68.1 \%$ versus $4 \mathrm{mg}$ in $31.1 \%$ (Table 2).

\section{Change from baseline in lipid profile}

Changes in lipid profiles at more than 8 weeks of pitavastatin treatment from baseline were analyzed (Table 3). The decrease in LDL-C was $-33.34 \pm 23.93,-39.13 \pm 39.46$, and $-46.27 \pm$ 
Table 1. Baseline Clinical Characteristics of Subjects

\begin{tabular}{|c|c|}
\hline Characteristic & No. $(\%)$ \\
\hline All patients & $28,343(100.0)$ \\
\hline \multicolumn{2}{|l|}{ Age, yr } \\
\hline$\geq 65$ & $10,803(38.1)$ \\
\hline$<65$ & $17,540(61.9)$ \\
\hline \multicolumn{2}{|l|}{ Gender } \\
\hline Male & $14,185(50.1)$ \\
\hline Female & $14,158(50.0)$ \\
\hline \multicolumn{2}{|c|}{ Use of lipid-lowering medications } \\
\hline Use of & $10,392(36.7)$ \\
\hline Stains & $9,864(34.8)$ \\
\hline Fibrates & $372(1.3)$ \\
\hline Other & $245(0.9)$ \\
\hline Non & $16,453(58.1)$ \\
\hline Unknown & $1,498(5.3)$ \\
\hline \multicolumn{2}{|l|}{ Liver disease } \\
\hline History of & $1,251(4.4)$ \\
\hline Fatty liver & $859(3.0)$ \\
\hline Hepatitis & $231(0.8)$ \\
\hline Other & $196(0.7)$ \\
\hline No & $26,700(94.2)$ \\
\hline Unknown & $392(1.4)$ \\
\hline \multicolumn{2}{|l|}{ Renal disease } \\
\hline History of & $944(3.3)$ \\
\hline Renal failure & $651(2.3)$ \\
\hline Pyelonephritis & $38(0.1)$ \\
\hline Nephrolithiasis & $31(0.1)$ \\
\hline Other & $254(0.9)$ \\
\hline No & $26,954(95.1)$ \\
\hline Unknown & $445(1.6)$ \\
\hline \multicolumn{2}{|l|}{ Comorbidity } \\
\hline History of & $19,102(67.4)$ \\
\hline Diabetes mellitus & $5,349(18.9)$ \\
\hline Hypertension & $11,030(38.9)$ \\
\hline Cardiovascular disease & $3,029(10.7)$ \\
\hline Cerebrovascular disease & 3,385 (11.9) \\
\hline Other & $4,811(17.0)$ \\
\hline No & $8,921(31.5)$ \\
\hline Unknown & $320(1.1)$ \\
\hline
\end{tabular}

$39.07 \mathrm{mg} / \mathrm{dL}$, respectively, after 1,2 , and $4 \mathrm{mg}$ pitavastatin treatment. After more than 8 weeks of treatment, there was a significant increase in HDL-C: $4.34 \pm 7.42,0.83 \pm 11.54$, and
Table 2. Administration of Pitavastatin

\begin{tabular}{lc}
\hline Variable & Value \\
\hline Duration of administration, day & $125.85 \pm 83.78$ \\
$<8 \mathrm{wk}$ & $442(1.6)$ \\
$\geq 8$ and $<16 \mathrm{wk}$ & $15,893(56.1)$ \\
$\geq 16$ and $<24 \mathrm{wk}$ & $6,400(22.6)$ \\
$\geq 24 \mathrm{wk}$ & $5,608(19.8)$ \\
Dose of administration & \\
$1 \mathrm{mg}$ & $146(0.5)$ \\
$2 \mathrm{mg}$ & $19,292(68.1)$ \\
$4 \mathrm{mg}$ & $8,802(31.1)$ \\
Other & $103(0.4)$ \\
\hline
\end{tabular}

Values are expressed as mean \pm standard deviation or number (\%).

$1.10 \pm 9.94 \mathrm{mg} / \mathrm{dL}$ in the 1,2 , and $4 \mathrm{mg}$ groups, respectively. After more than 8 weeks of treatment, there was a significant decrease in triglyceride: $-27.93 \pm 61.22,-26.16 \pm 109.96$, and $-40.54 \pm 213.27 \mathrm{mg} / \mathrm{dL}$ in the 1,2 , and $4 \mathrm{mg}$ groups, respectively (Table 3).

To evaluate the lipid lowering efficacy of pitavastatin in patients who had not been taken statin previously, we analyzed the $\%$ changes in lipid profiles depending on whether lipid-lowering medications have been administered prior to study or not (Supplemental Table S1). The \% change of total cholesterol in patients who have not been treated with lipid lowering medications including statin, fibrate and omega-3 fatty acid prior to study was $-21.1 \%$ in the $1 \mathrm{mg}$ group, $-22.5 \%$ in the $2 \mathrm{mg}$ group, and $-27.3 \%$ in the $4 \mathrm{mg}$ group. The $\%$ change of LDL-C in those patients was $-23.4 \%,-29.1 \%$, and $-35.2 \%$ in the 1,2 , and $4 \mathrm{mg}$ groups, respectively; the decrease was significant and dose dependent with pitavastatin (Fig. 2). Meanwhile, the \% change of LDL-C in patients who had been administered other statins, fibrates or others prior to study was $-16.1 \%,-8.5 \%$, and $-16.7 \%$ in the 1,2 , and $4 \mathrm{mg}$ groups, respectively (Supplemental Table S1). The \% change of HDL-C in patients who have not been treated with lipid lowering medications prior to study was $12.1 \%, 5.2 \%$, and $4.1 \%$ in the 1,2 , and $4 \mathrm{mg}$ groups, respectively. The $\%$ change of triglyceride in patients who have not been treated with lipid lowering medications prior to study was $-10.5 \%,-7.3 \%$, and $-9.1 \%$ in the 1,2 , and $4 \mathrm{mg}$ groups, respectively (Fig. 2).

\section{Change from baseline in biochemical profiling of safety}

During the study period, laboratory test results were collected. 


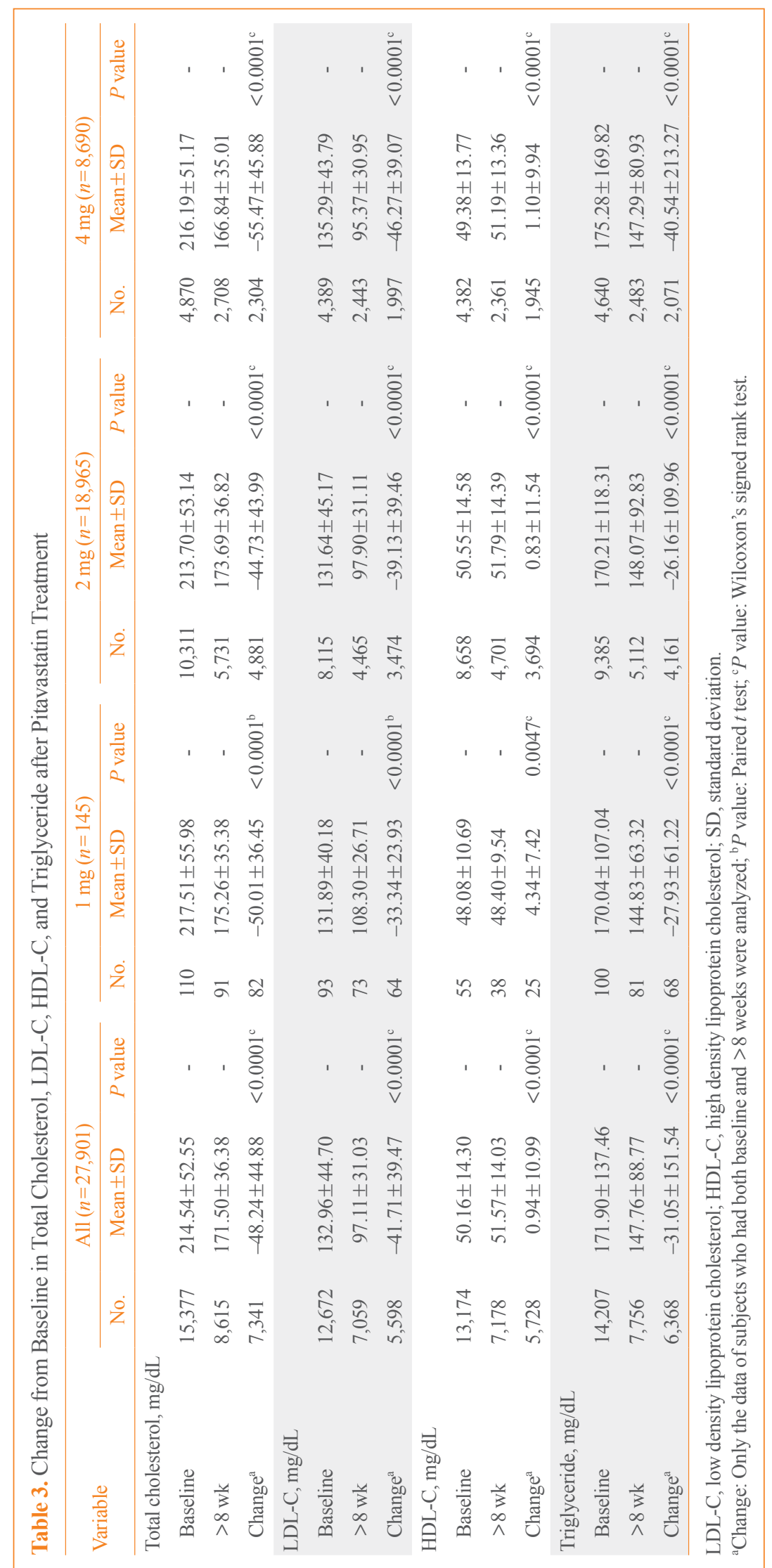



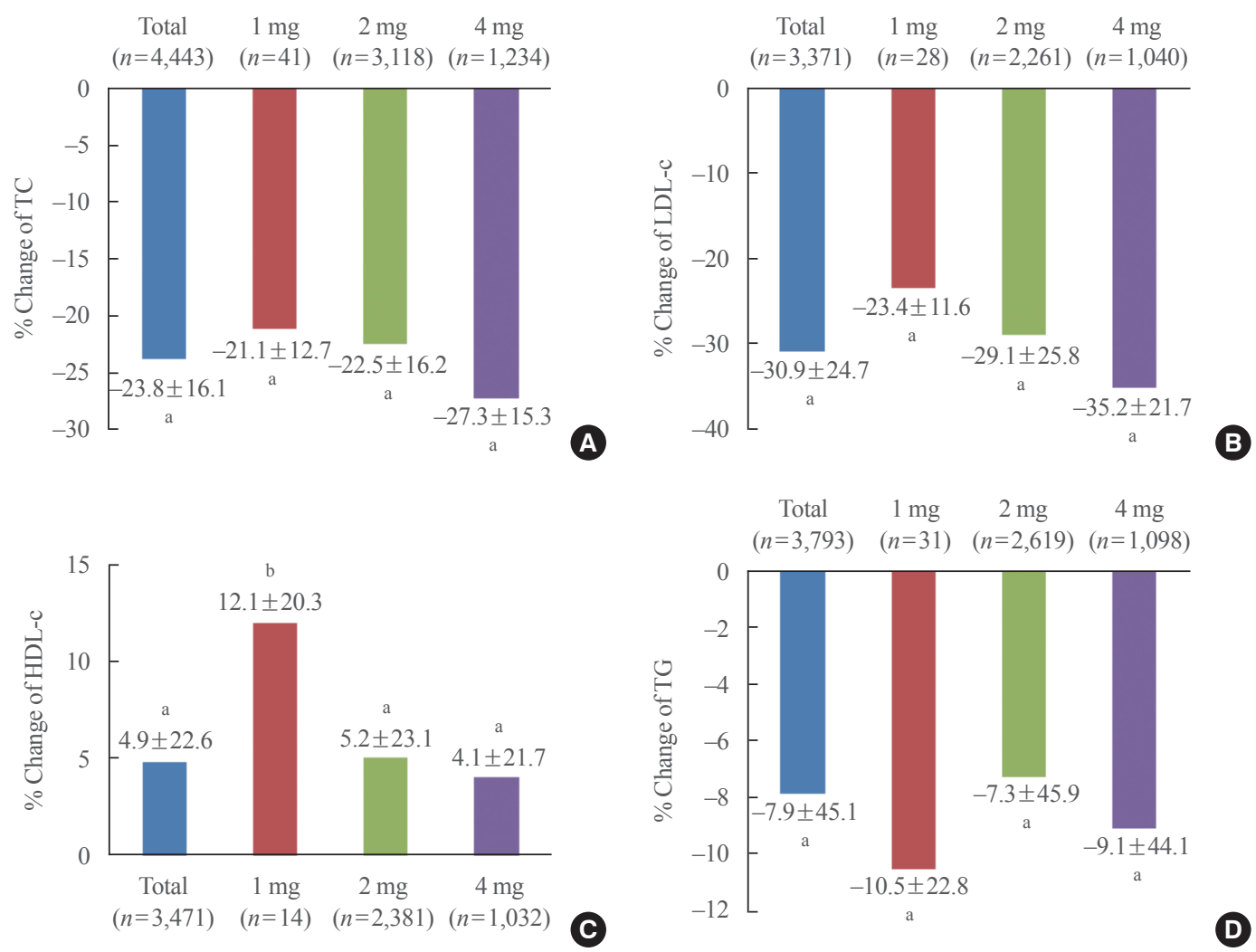

Fig. 2. \% Change of lipid profile from baseline in patients who have not been treated with lipid lowering medications prior to study. (A) Total cholesterol (TC), (B) and low density lipoprotein cholesterol (LDL-C), (C) high density lipoprotein cholesterol (HDL-C), and (D) triglyceride (TG). ${ }^{\mathrm{a}} P<0.001 ;{ }^{\mathrm{b}} P<0.05$.

Supplemental Table S2 shows the biochemical profiling results from peripheral blood for safety assessment. Number of subjects experiencing, upon pitavastatin treatment, Grade 2 toxicity according to Common Terminology Criteria for Adverse Events (CTCAE) v4.03 [18] was counted since baseline. Regarding liver-related safety data, ALT and AST levels were not elevated higher than those of previously reported studies. In terms of the myopathy, there was no increase in abnormalities compared to previous reports (Supplemental Table S2).

\section{Adverse event and adverse drug reaction}

Fig. 3 shows the safety results of pitavastatin in 28,343 patients with hyperlipidemia (Supplemental Table S3) and Fig. 4 shows the results of musculoskeletal system disorders, known statinassociated adverse effects (Supplemental Table S4). A total of 492 patients (1.74\%) reported AEs. Of those, 123 patients $(0.43 \%)$ experienced drug related ADRs. As shown in Figs. 3, 4, administration of pitavastatin was tolerable.

\section{DISCUSSION}

Although RCTs provide useful information about the efficacy and safety of drugs, they do not always reflect the real world. In addition, there might be differences between Asian and Western populations, or even between the Japanese and Koreans within the Asian population.

In this large-scale PROOF study in Koreans, the $\%$ change of LDL-C indicated a dose dependent reduction: $-23.4 \%,-29.1 \%$, and $-35.2 \%$ in the 1,2 , and $4 \mathrm{mg}$ groups, respectively in patients who have not been treated with lipid lowering medications prior to study. However, the \% change of LDL-C by dose reported in pitavastatin pivotal studies was approximately $-31 \%,-39 \%$, and $-44 \%$ in the 1,2 , and $4 \mathrm{mg}$ groups, respectively. These results satisfied the definition of moderate-intensity statin with $30 \%$ to $50 \%$ LDL-C lowering effect according to the American College of Cardiology/American Heart Association (AHA/ACC) guideline published in 2013 [16,19]. At the lower $\%$ change of LDL-C in the present study, the $2 \mathrm{mg}$ group showed an effect near to the definition of moderate-intensity 


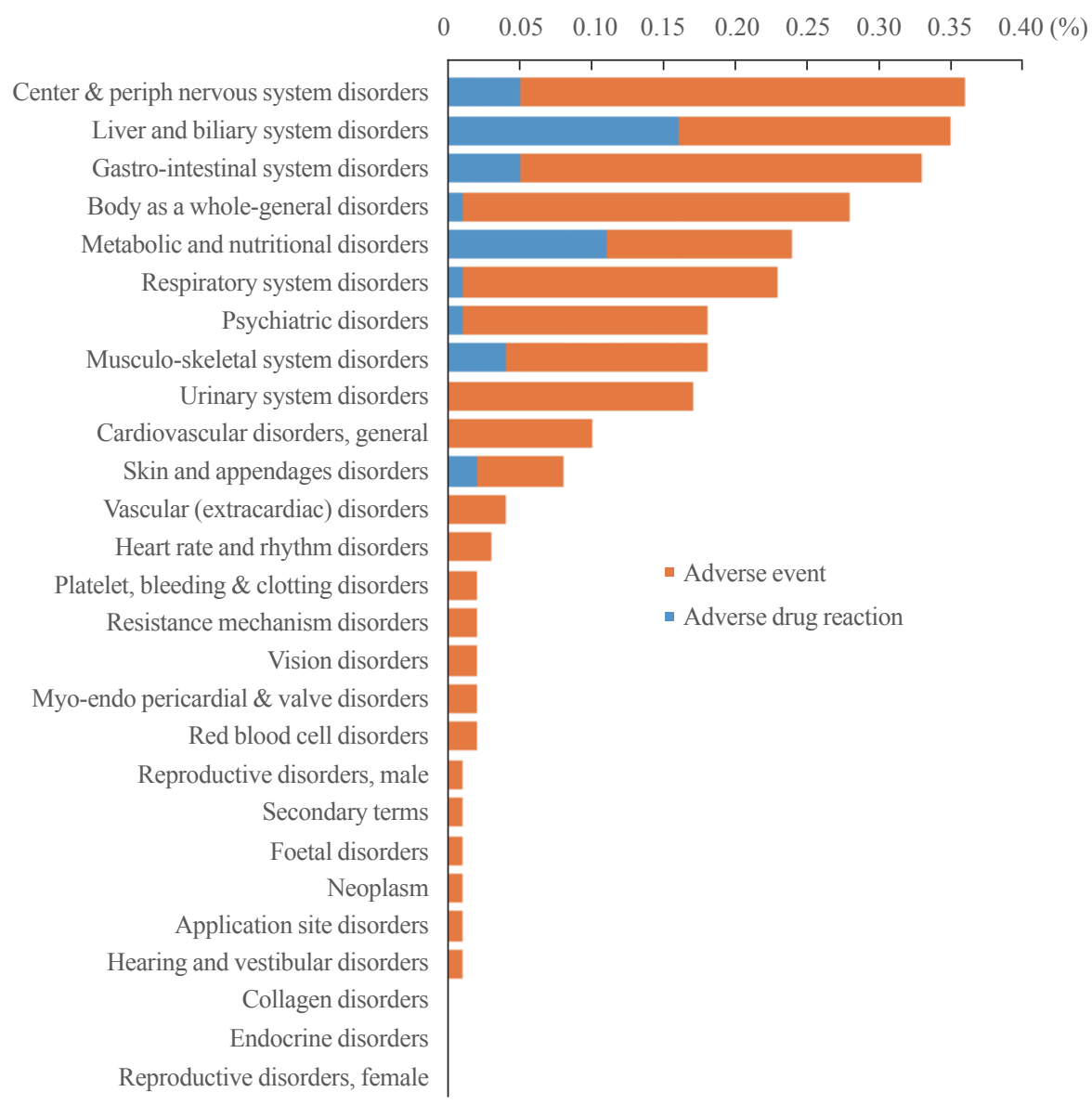

Fig. 3. All adverse events and adverse drug reactions of pitavastatin treatment.

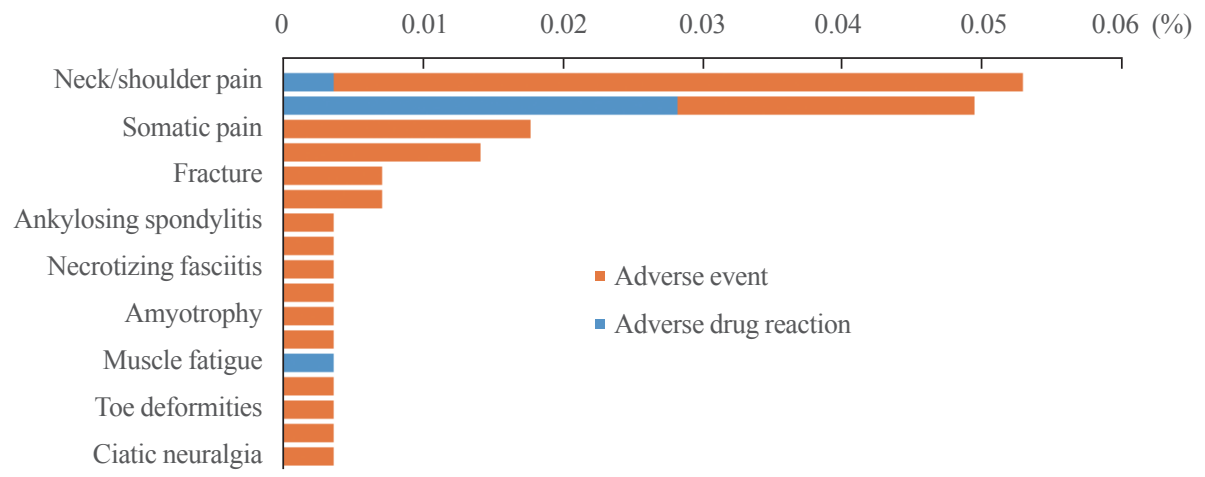

Fig. 4. Musculoskeletal disorders after pitavastatin treatment.

statin, and the $4 \mathrm{mg}$ group showed an effect that satisfies the definition. This is thought to be attributable to the limitation of the observational study with lower compliance to prescribed medications. In addition, the LIVES study in Japanese patients reported a 31.3\% reduction in LDL-C at week 104 of pitavastatin treatment, which is comparable to the outcome in Korea after mean 18 weeks of treatment. In addition, the reduction in $\%$ change of LDL-C was decreased in patients who had been administered other statins, fibrates or others in the PROOF study. This is because baseline LDL-C levels were already lower than those of patients who had never been administered lipidlowering medications. In the Japanese patients, HDL-C levels 
were up 5.9\%, while in Koreans, there was an $11.7 \%$, 4.2\%, and $4.4 \%$ increase in HDL-C levels in the 1,2 , and $4 \mathrm{mg}$ groups, respectively [17].

The most common adverse effects relevant to statins are abnormal hepatic function and myopathy. Unlike other statins, pitavastatin is highly hydrophilic, is extensively excreted in the bile, and is not metabolized by cytochrome P450 (particularly not by CYP3A4). A previous study reported a more frequent increase in liver enzyme levels by statins in a high-intensity statin group with an incidence of $2 \%$ to $3 \%$ versus $<1 \%$ in a mediumintensity statin group [20]. In comparison, the incidence of liver and biliary system disorders was $0.35 \%$ in the pitavastatin group. In the present study, the incidence of abnormal liver function caused by pitavastatin was $0.16 \%$, which is somewhat higher than $0.04 \%$ reported in the LIVES study in Japan, but comparable to other previous reports.

The incidence of AEs of musculoskeletal system disorders was $10 \%$ to $29 \%$ in observational studies and $1 \%$ to $2 \%$ in RCTs [21-23]. The present study, in comparison, showed a low incidence of $0.18 \%$. Despite a markedly low incidence of muscular adverse symptoms caused by statins, it is also reported that the musculoskeletal disorders such as myopathy and rhabdomyolysis may lead to acute renal failure as a life-threatening secondary complication [9]. HMG-CoA reductase inhibitors reduce cholesterol production thereby interfering with the formation and maintenance of cellular membranes and reducing coenzyme-Q and heme-A produced from cholesterol precursors. Coenzyme-Q, one of the antioxidants in humans, plays a significant role in the creation of adenosine triphosphate along with heme-A. The reduced production of these biomolecules is deemed to be the cause of toxicities of HMG-CoA reductase inhibitors [24-27].

Among the musculoskeletal adverse effects caused by statin, the most common symptom is myalgia, and the severest one is rhabdomyolysis [28]. In the United States, myalgia is a common cause of treatment withdrawal; more than $60 \%$ of patients who stopped statin treatment reported musculoskeletal adverse effects [29]. In the PROOF study in Koreans, however, the incidence of myalgia was $0.03 \%$ (eight subjects) among 28,343 subjects, which was very low, and there were no reports of rhabdomyolysis. In addition, the incidence of ADRs assessed to be caused by pitavastatin treatment was $0.43 \%$ (123/28,343). In the LIVES study in Japanese patients, ADRs were reported in $10.4 \%$ of the study subjects, and of those, myalgia occurred to $1.1 \%$. Compared to these figures, the incidence in Koreans is deemed low [17].
This study has three main limitations. The first is that this trial is an observation study. Due to this intrinsic limitation, many factors, such as treatment group assignment, compliance, baseline characteristic and etc. that may affect the results could not be controlled to minimize the bias. However, the results of this study have significance that they reflect the real world in which real patients take pitavastatin. The second was that the same number of subjects could not be assigned according to the dosage of pitavastatin. The number of subjects in the pitavastatin 1 mg group was very small compared to the other 2 or $4 \mathrm{mg}$ groups, and dose dependent results could not be confirmed in the HDL-C and TG. In other RCTs that confirmed the lipid profile of each dose of pitavastatin, it was reported that HDL-C increased and TG decreased depending on dosage $[8,16]$. It seems to have had a greater influence on the results, since it is known that diet or alcohol has a greater influence on HDL-C and TG, and the number of analysis cases in the pitavastatin $1 \mathrm{mg}$ group was 30 to 70 times less than the other groups [30,31]. At last, this study only collected safety information on items known to cause abnormal levels when taking pitavastatin, and did not collect fasting glucose or glycosylated haemoglobin data. Previously, statins were reported to increase the risk of new onset DM, and this was considered a class effect [32,33]. However, some studies also showed that pitavastatin has no deleterious effect on the glucose deterioration [34-36]. It is necessary to compare glucose alteration and the risk of new onset DM between pitavastatin and other statins through additional RCT in the future.

In conclusion, this observational study has demonstrated that Livalo (pitavastatin) Tab. lowers LDL-C in a dose dependent manner (1, 2, $4 \mathrm{mg})$ and can be safely used without the risk of abnormal hepatic function or musculoskeletal adverse effects.

\section{CONFLICTS OF INTEREST}

This study was funded by the JW Pharmaceutical Corporation.

\section{AUTHOR CONTRIBUTIONS}

Conception or design: S.R.K. Acquisition, analysis, or interpretation of data: I.K.J., S.R.K. Drafting the work or revising: I.K.J., S.R.K. Final approval of the manuscript: I.K.J., S.R.K.

\section{ORCID}

In-Kyung Jeong https://orcid.org/0000-0001-7857-546X

Sung-Rae Kim https://orcid.org/0000-0001-6417-8412 


\section{REFERENCES}

1. World Health Organization. Cardiovascular diseases (CVDs) [Internet]. Geneva: WHO; 2017 [cited 17 May 2017]. Available from: https://www.who.int/news-room/fact-sheets/detail/ cardiovascular-diseases-(cvds).

2. Rhee EJ, Kim HC, Kim JH, Lee EY, Kim BJ, Kim EM, et al. 2018 Guidelines for the management of dyslipidemia. Korean J Intern Med 2019;34:723-71.

3. Saito Y, Teramoto T, Yamada N, Itakura H, Hata Y, Nakaya $\mathrm{N}$, et al. Clinical efficacy of NK-104 (pitavastatin), a new synthetic HMG-CoA reductase inhibitor, in the dose finding, double blind, three-group comparative study. J Clin Therap Med 2001;17:829-55.

4. Collins R, Reith C, Emberson J, Armitage J, Baigent C, Blackwell L, et al. Interpretation of the evidence for the efficacy and safety of statin therapy. Lancet 2016;388:2532-61.

5. Ose L, Budinski D, Hounslow N, Arneson V. Comparison of pitavastatin with simvastatin in primary hypercholesterolaemia or combined dyslipidaemia. Curr Med Res Opin 2009; 25:2755-64.

6. Kwon JE, Kim Y, Hyun S, Won H, Shin SY, Lee KJ, et al. Cholesterol lowering effects of low-dose statins in Korean patients. J Lipid Atheroscler 2014;3:21-8.

7. Lee SH, Chung N, Kwan J, Kim DI, Kim WH, Kim CJ, et al. Comparison of the efficacy and tolerability of pitavastatin and atorvastatin: an 8-week, multicenter, randomized, open-label, dose-titration study in Korean patients with hypercholesterolemia. Clin Ther 2007;29:2365-73.

8. Taguchi I, Iimuro S, Iwata H, Takashima H, Abe M, Amiya E, et al. High-dose versus low-dose pitavastatin in Japanese patients with stable coronary artery disease (REAL-CAD): a randomized superiority trial. Circulation 2018;137:1997-2009.

9. Newman CB, Preiss D, Tobert JA, Jacobson TA, Page RL 2nd, Goldstein LB, et al. Statin safety and associated adverse events: a scientific statement from the American Heart Association. Arterioscler Thromb Vasc Biol 2019;39:e3881.

10. De Alava E, Sola JJ, Lozano MD, Pardo-Mindan FJ. Rhabdomyolysis and acute renal failure in a heart transplant recipient treated with hypolipemiants. Nephron 1994;66:242-3.

11. Fernandez Zatarain G, Navarro V, Garcia H, Villatoro J, Calvo C. Rhabdomyolysis and acute renal failure associated with lovastatin. Nephron 1994;66:483-4.

12. Farmer JA, Torre-Amione G. Comparative tolerability of the HMG-CoA reductase inhibitors. Drug Saf 2000;23:197-
213.

13. Manoukian AA, Bhagavan NV, Hayashi T, Nestor TA, Rios C, Scottolini AG. Rhabdomyolysis secondary to lovastatin therapy. Clin Chem 1990;36:2145-7.

14. Pfizer Limited. Atorvastatin $10 \mathrm{mg}$ Film Coated Tablets: Summary of Product Characteristics (SmPC) [Internet]. The electronic medicines compendium (emc); 2019 [cited 2020 Oct 29]. Available from: https://www.medicines.org.uk/emc/ product/4109/smpc\#gref.

15. Teramoto T. Pitavastatin: clinical effects from the LIVES Study. Atheroscler Suppl 2011;12:285-8.

16. Betteridge J. Pitavastatin: results from phase III \& IV. Atheroscler Suppl 2010;11:8-14.

17. Yokote K, Shimano H, Urashima M, Teramoto T. Efficacy and safety of pitavastatin in Japanese patients with hypercholesterolemia: LIVES study and subanalysis. Expert Rev Cardiovasc Ther 2011;9:555-62.

18. National Cancer Institute. Common Terminology Criteria for Adverse Events (CTCAE) v4.03. Bethesda: U.S. Department of Health and Human Services, National Institutes of Health; 2010.

19. Stone NJ, Robinson JG, Lichtenstein AH, Bairey Merz CN, Blum CB, Eckel RH, et al. 2013 ACC/AHA guideline on the treatment of blood cholesterol to reduce atherosclerotic cardiovascular risk in adults: a report of the American College of Cardiology/American Heart Association Task Force on Practice Guidelines. J Am Coll Cardiol 2014;63(25 Pt B):2889-934.

20. Cohen DE, Anania FA, Chalasani N; National Lipid Association Statin Safety Task Force Liver Expert Panel. An assessment of statin safety by hepatologists. Am J Cardiol 2006;97(8A):77C-81C.

21. Bruckert E, Hayem G, Dejager S, Yau C, Begaud B. Mild to moderate muscular symptoms with high-dosage statin therapy in hyperlipidemic patients: the PRIMO study. Cardiovasc Drugs Ther 2005;19:403-14.

22. Davidson MH, Clark JA, Glass LM, Kanumalla A. Statin safety: an appraisal from the adverse event reporting system. Am J Cardiol 2006;97(8A):32C-43C.

23. Adhyaru BB, Jacobson TA. Safety and efficacy of statin therapy. Nat Rev Cardiol 2018;15:757-69.

24. Sylvain-Moore H, Worden JP Jr. Lovastatin-associated rhabdomyolysis. Heart Lung 1991;20(5 Pt 1):464-6.

25. Waclawik AJ, Lindal S, Engel AG. Experimental lovastatin myopathy. J Neuropathol Exp Neurol 1993;52:542-9.

26. Wallace CS, Mueller BA. Lovastatin-induced rhabdomyoly- 
sis in the absence of concomitant drugs. Ann Pharmacother 1992;26:190-2.

27. Hume AL. Comment: lovastatin-induced rhabdomyolysis in the absence of concomitant drugs. Ann Pharmacother 1992; 26:1303.

28. Pedro-Botet J, Nunez-Cortes JM, Flores JA, Rius J. Muscle symptoms related with statin therapy in general practice. Atherosclerosis 2015;241:e197.

29. Khan A, Maki KC, Ito MK, Cohen JD, Sponseller CA, Bell $\mathrm{M}$, et al. Statin associated muscle symptoms: characteristics of patients and recommendations by providers. J Clin Lipidol 2015;9:460.

30. Crouse JR, Grundy SM. Effects of alcohol on plasma lipoproteins and cholesterol and triglyceride metabolism in man. J Lipid Res 1984;25:486-96.

31. Wakabayashi I. Alcohol intake and triglycerides/high-density lipoprotein cholesterol ratio in men with hypertension. Am J Hypertens 2013;26:888-95.

32. Ridker PM, Danielson E, Fonseca FA, Genest J, Gotto AM
$\mathrm{Jr}$, Kastelein JJ, et al. Rosuvastatin to prevent vascular events in men and women with elevated C-reactive protein. N Engl J Med 2008;359:2195-207.

33. Betteridge DJ, Carmena R. The diabetogenic action of statins: mechanisms and clinical implications. Nat Rev Endocrinol 2016;12:99-110.

34. Choi SH, Lim S, Hong ES, Seo JA, Park CY, Noh JH, et al. PROPIT: a PROspective comparative clinical study evaluating the efficacy and safety of PITavastatin in patients with metabolic syndrome. Clin Endocrinol (Oxf) 2015;82:670-7.

35. Jeong HS, Hong SJ, Son S, An H, Kook H, Joo HJ, et al. Incidence of new-onset diabetes with $1 \mathrm{mg}$ versus $4 \mathrm{mg}$ pitavastatin in patients at high risk of developing diabetes during a 3-year follow-up. Cardiovasc Diabetol 2019;18:162.

36. Choi JY, Choi CU, Hwang SY, Choi BG, Jang WY, Kim DY, et al. Effect of pitavastatin compared with atorvastatin and rosuvastatin on new-onset diabetes mellitus in patients with acute myocardial infarction. Am J Cardiol 2018;122:922-8. 